\title{
A Chlorogenic Acid Mediated Synthesis of Silver Nanoparticles and Their Application for the Antibacterial Functionalization of Silk Fiber
}

\author{
Ling-Ling $\mathrm{Li}^{\mathrm{a}}$ and Ren-Cheng Tang ${ }^{\mathrm{b}}$ \\ College of Textile and Clothing Engineering, Soochow University, Suzhou 215123, China \\ a1309027860@qq.com, btangrencheng@suda.edu.cn
}

\begin{abstract}
A chlorogenic acid mediated synthesis of silver nanoparticles (AgNPs) from silver nitrate solution was investigated, and the preparation conditions of AgNPs were discussed. AgNPs could be rapidly prepared in a weakly alkaline condition, and the resulting AgNPs had an average size of about $35 \mathrm{~nm}$. When AgNPs were applied to functionalize silk fiber, a weakly acidic condition could provide a high adsorption quantity of Ag loading. The distribution of AgNPs on silk fiber was confirmed by the scanning electron microscope and X-ray diffraction analyses. The functionalized silk fiber exhibited excellent antibacterial activity against Escherichia coli and Staphylococcus aureus.
\end{abstract}

Keywords: silk, silver nanoparticles, chlorogenic acid, functionalization, antibacterial activity.

\section{Introduction}

Silk fiber has found a series of applications in textile and medical fields due to its excellent properties such as softness, gentle luster, comfortableness, hygroscopicity, biocompatibility and non-toxicity [1]. However, silk fiber suffers from some shortcomings such as wrinkling, photooxidation, deterioration, yellowing, poor antibacterial activity, and poor UV protection capability [1, 2]. In particular, because of its protein and moisture-adsorbing nature, silk fiber allows microbial growth and multiplication which give rise to foul odors, mildew formation, discoloration, fiber degradation, and disease infection. Therefore, the antibacterial performance of silk fiber should be upgraded. A series of antibacterial agents including quaternary ammonium compounds, quaternary ammonium organosilanes, chlorinated compounds, inorganic materials, chitosan, antimicrobial peptides, bactericidal enzyme lysozyme, and natural dyes have been applied to the antibacterial functionalization of silk fiber [1-3]. Out of these antibacterial agents, silver nanoparticles (AgNPs) have drawn great attraction due to their excellent antibacterial function. Various chemical and physical approaches have been used to prepare AgNPs. In recent years, the green synthesis of AgNPs using plant extracts has become valuable alternatives to chemical synthesis. Honeysuckle, the flower of Lonicera japonica Thunb, is one of the most famous traditional Chinese medicinal herbs used for the treatment of common cold and fever. Chlorogenic acid (CA) is the major bioactive compound in honeysuckle. In this work, CA was employed as reductant and stabilizer for the synthesis of AgNPs from silver nitrate, and the preparation conditions of AgNPs were studied. Subsequently, the resulting AgNPs were applied to the antibacterial functionalization of silk fiber.

\section{Materials and Methods}

\subsection{Materials}

Silver nitrate and chlorogenic acid (CA) were bought from Sinopharm Chemical Reagent Co. Ltd, Shanghai,China, and Changsha Staherb Natural Ingredients Co. Ltd., China. The silk fabric of crepe de chine was purchased from Suzhou Jiaduoli Silk Apparel Co. Ltd., China.

\subsection{Preparation of AgNPs}

The preparation of AgNPs was performed in the sealed and conical flasks immersed in a constant temperature bath. The $\mathrm{pH}$ of the mixture solution of $\mathrm{CA}$ and $\mathrm{AgNO}_{3}$ was adjusted by acetic acid and sodium carbonate. The mixture solution was stirred for a desired time at a constant temperature.

To study the effect of $\mathrm{pH}$, the $\mathrm{pH}$ of the solution was adjusted to several different values, and the temperature, time, $\mathrm{AgNO}_{3}$ dosage, and CA dosage were $70{ }^{\circ} \mathrm{C}, 60 \mathrm{~min}, 1.0 \mathrm{mmol} / \mathrm{L}$, and $0.2 \mathrm{~g} / \mathrm{L}$, 
respectively. To determine the effect of temperature, six temperatures $\left(40,50,60,70,80\right.$ and $\left.90{ }^{\circ} \mathrm{C}\right)$ were used, and the other parameters were: $\mathrm{AgNO}_{3} 1 \mathrm{mmol} / \mathrm{L}, \mathrm{CA} 0.2 \mathrm{~g} / \mathrm{L}, \mathrm{pH} 10$, and $60 \mathrm{~min}$. To estimate the effect of time, the mixture solution was stirred for different times $(5,10,20,40,60$ and $80 \mathrm{~min}$ ), and the other parameters were: $\mathrm{AgNO}_{3} 1 \mathrm{mmol} / \mathrm{L}, \mathrm{CA} 0.2 \mathrm{~g} / \mathrm{L}, \mathrm{pH} 10$, and $70{ }^{\circ} \mathrm{C}$. To study the effect of CA dosage, five CA dosages $(0.1,0.2,0.3,0.4$ and $0.5 \mathrm{~g} / \mathrm{L})$ were employed, and the other parameters were as follows: $\mathrm{AgNO}_{3} 1 \mathrm{mmol} / \mathrm{L}, \mathrm{pH} 10,70{ }^{\circ} \mathrm{C}$, and $60 \mathrm{~min}$. To evaluate the effect of $\mathrm{AgNO}_{3}$ dosage, seven $\mathrm{AgNO}_{3}$ dosages ranging from 0.2 to $2.0 \mathrm{mmol} / \mathrm{L}$ were employed, and the other parameters were as follows: CA $0.2 \mathrm{~g} / \mathrm{L}, \mathrm{pH} 10,70{ }^{\circ} \mathrm{C}$, and $60 \mathrm{~min}$.

\subsection{Application of AgNPs}

The AgNPs dispersion was prepared in the following conditions: $\mathrm{AgNO}_{3} 1.0 \mathrm{mmol} / \mathrm{L}, \mathrm{CA} 0.4 \mathrm{~g} / \mathrm{L}$, $\mathrm{pH} 10,70{ }^{\circ} \mathrm{C}$, and $60 \mathrm{~min}$. After the $\mathrm{pH}$ values of the prepared AgNPs were adjusted to $4-7.7$ by acetic acid, the AgNPs were applied to treat silk fabrics. The treatment started at $30^{\circ} \mathrm{C}$, the temperature was raised at a rate of $2{ }^{\circ} \mathrm{C} / \mathrm{min}$ up to $80^{\circ} \mathrm{C}$, and at this temperature the treatment continued for $60 \mathrm{~min}$. The sample treated using AgNPs at $\mathrm{pH} 4$ was used for the measurements of antibacterial properties.

\subsection{Measurements}

The UV-vis spectroscopic measurement of AgNPs solution was carried out on a Shimadzu UV-1800 UV-vis spectrophotometer (Shimadzu Co., Japan). The average particle size and zeta potential of AgNPs were measured using a Zetasizer Nano ZS 90 (Malvern Instruments Ltd, UK). The X-ray diffraction (XRD) measurement of AgNPs powder and silk fiber was performed on a X'Pert-Pro MPD X-ray diffractometer (PANalytical B.V., Almelo, Netherlands). The surface morphology of silk fiber was observed using a Hitachi-S4800 field-emission scanning electron microscope (SEM) (Hitachi High Technologies America Inc., USA). The apparent color depth (K/S) and color difference (delta E) of silk fabric was evaluated by a HunterLab UltraScan PRO reflectance spectrophotometer. The antibacterial activity of silk fabric against Escherichia coli (E. coli) and Staphylococcus aureus (S. aureus) was evaluated according to GB/T 20944.3-2008.

\section{Results and Discussion}

\subsection{Preparation of AgNPs}

Because AgNPs exhibit strong absorption band in the visible light region due to the surface plasmon resonance (SPR), the formation of AgNPs can be monitored by the UV-vis absorption spectroscopy. In this study, the effects of $\mathrm{pH}$, temperature, time, and $\mathrm{AgNO}_{3}$ dosage, and CA dosage employed in the preparation of AgNPs were discussed.

Fig. 1 shows the UV-vis absorption spectra of AgNPs. The SPR peak varied with the changed conditions of AgNPs preparation, and ranged from 410 to $425 \mathrm{~nm}$. Among five factors, the reaction time had the smallest effect on the absorbance at the maximum absorption wavelength (Fig. 1c), implying that the formation of AgNPs is rapid. The intensity of the SPR peak increased gradually with increasing $\mathrm{pH}$ up to 9.75, and then decreased slightly with further increasing pH (Fig. 1a). This indicates that an alkaline condition is more conducive to the preparation of AgNPs, possibly due to the enhanced reducing power of CA with increasing $\mathrm{pH}$. When temperature was higher than $60{ }^{\circ} \mathrm{C}$, the intensity of the SPR peak increased obviously (Fig. 1b), revealing that a higher temperature is imperative for the reduction of Ag ions. As expected, the absorbance of the SPR peak increased with an increase in CA and $\mathrm{AgNO}_{3}$ dosage (Figs. 1d and 1e).

The particle size distribution and zeta potential of the prepared AgNPs were determined by the dynamic light scattering analysis. As shown in Figs. 2a and 2b, the average size was about $35 \mathrm{~nm}$, and the zeta potential was about $-24 \mathrm{mV}$. Additionally, a low polydispersity index was also found, revealing the uniform size distribution of AgNPs. Fig. 2c shows four characteristic crystalline diffraction peaks of AgNPs powder at $2 \theta$ of $38.0^{\circ}, 44.2^{\circ}, 65.1^{\circ}$, and $77.3^{\circ}$ corresponding to the diffraction of the (111), (200), (220), and (311) lattice planes. The diffraction peaks are consistent with the standard patterns of crystal silver, indicating a face-centered-cube phase of the Ag crystal [4]. 

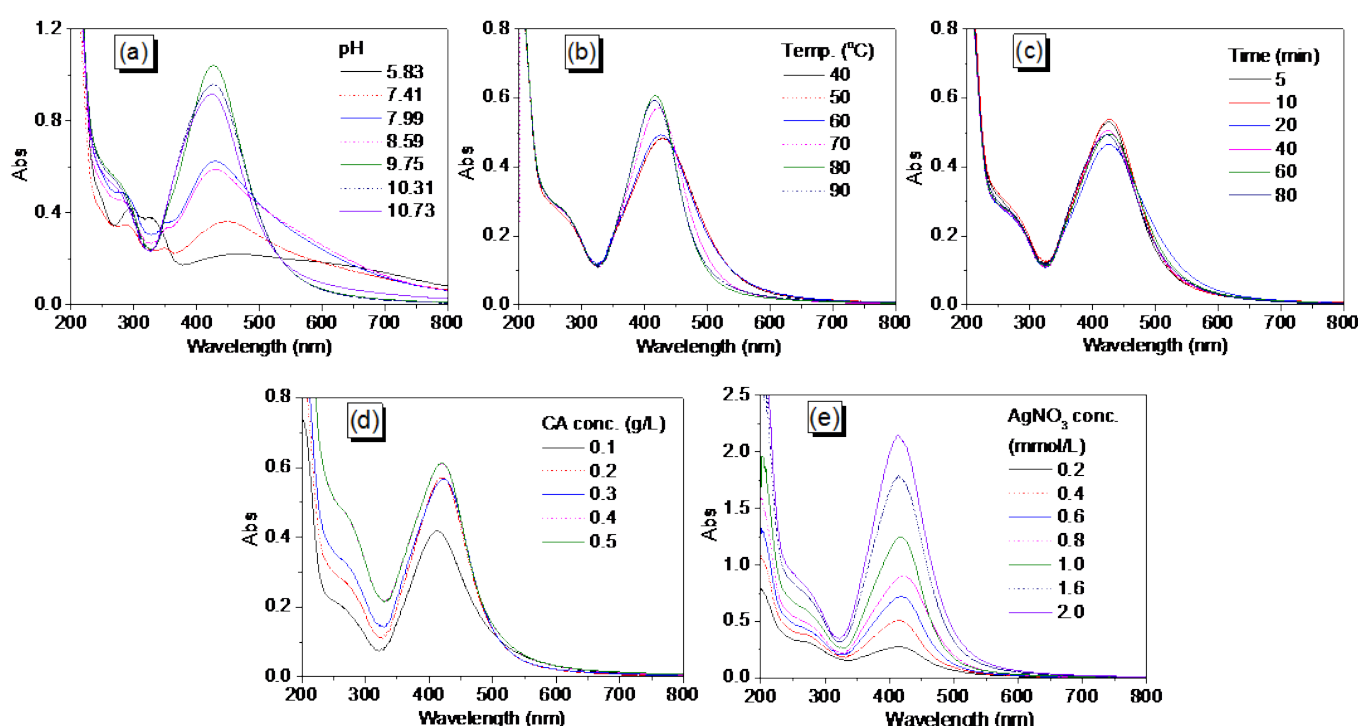

Fig. 1 Dependence of the UV-vis absorption spectra of AgNPs dispersion on $\mathrm{pH}$ (a), temperature (b), time (c), CA dosage (d), and $\mathrm{AgNO}_{3}$ dosage (e)
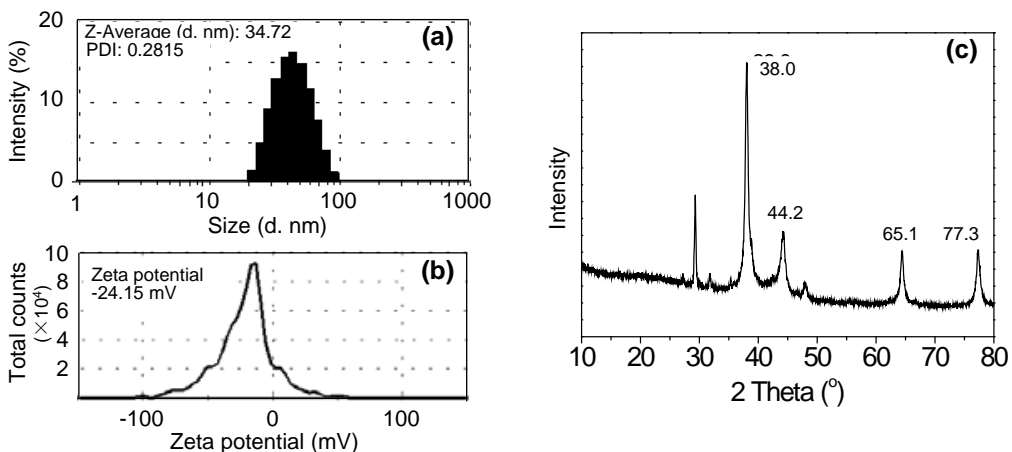

Fig. 2 Particle size distribution (a), zeta potential (b) and XRD (c) of AgNPs

\subsection{Application of AgNPs to the Functionalization of Silk Fiber}

The AgNPs dispersion was prepared in the following conditions: $\mathrm{AgNO}_{3} 1.0 \mathrm{mmol} / \mathrm{L}, \mathrm{CA} 0.4 \mathrm{~g} / \mathrm{L}$, $\mathrm{pH} 10,70^{\circ} \mathrm{C}$, and $60 \mathrm{~min}$. When the prepared AgNPs dispersion was used to treat silk fabric, its $\mathrm{pH}$ was discussed as the most important parameter in terms of the color depth and color difference of silk fabric. Fig. 3a shows that K/S and Delta E decreased as $\mathrm{pH}$ increased from 4 to 7.7, suggesting that silk fiber has a better adsorption capacity for AgNPs in weakly acidic conditions. This can be explained by the increased attraction forces between the negatively charged AgNPs and the positively charged silk fiber in weakly acidic conditions. It is well known that silk fabric has poor antibacterial function due to its protein nature. Fig. 3b shows that the antibacterial activity of the Ag-loaded fabric against E. coli and S. aureus was $99.21 \%$ and $99.01 \%$, respectively. This illustrates that the incorporation of AgNPs imparts excellent antibacterial ability to silk fabric. Although the antibacterial activity decreased with increasing wash cycles, it still retained a high value after 20 washing cycles, indicating that the antibacterial function of the treated fabric has good durability to washing.

The surface morphology of the silk fibers treated without and with AgNPs was observed by SEM. From Fig. 4a, it is clear that compared with the clean and smooth surface of the untreated silk fiber, the surface of the treated fiber was covered with a large number of tiny Ag particles. The surface inspection indicates the successful incorporation of AgNPs on silk fiber. The incorporation of AgNPs was also confirmed by the XRD pattern shown in Fig. 4b. No significant changes of the crystalline diffraction peaks were found for the untreated and treated silk fibers, suggesting that the AgNPs treatment does not alter the crystalline structure of silk fiber. For the AgNPs treated silk fiber, two new minor diffraction peaks appeared at $2 \theta=38.0^{\circ}$ and $44.2^{\circ}$, which are the characteristic crystalline 
diffraction peaks of AgNPs. This finding also confirms that the AgNPs with excellent crystalline structure are distributed on the treated silk fiber.
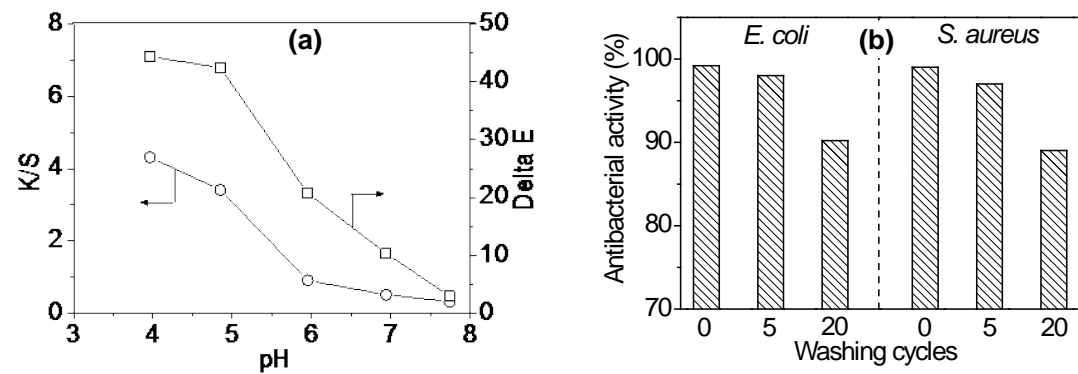

Fig. $3 \mathrm{PH}$ dependence of AgNPs treatment (a), and antibacterial activity of the functionalized silk fabric (b)

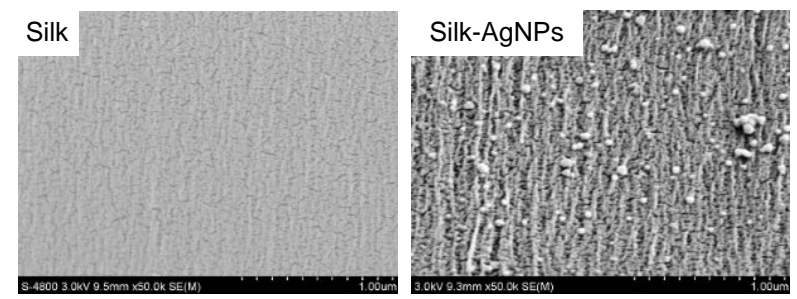

(a) SEM

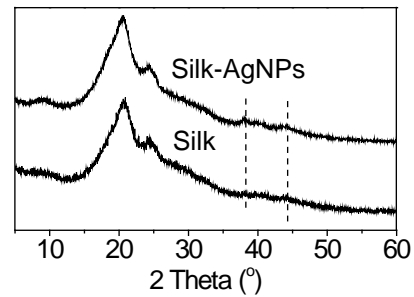

(b) XRD

Fig. 4 SEM images (a), and XRD patterns (b) of the silk fibers without and with functionalization

\section{Conclusions}

In this work, AgNPs were first prepared using $\mathrm{CA}$ and $\mathrm{AgNO}_{3}$, and then applied for the antibacterial functionalization of silk fabric. When CA was used as a reducing agent, AgNPs were rapidly prepared in an alkaline condition and at $70{ }^{\circ} \mathrm{C}$. The resulting AgNPs had an average size of about 35 nm. The application of AgNPs for the antibacterial treatment of silk fabric at $\mathrm{pH} 4$ could provide a high adsorption quantity of AgNPs. The loading of AgNPs on silk fiber was confirmed by SEM and XRD. The loading of AgNPs imparted excellent antibacterial activity against $E$. coli and $S$. aureus to silk fabric, and furthermore, the antibacterial function of the treated fabric had good durability to washing.

\section{Acknowledgments}

This study was funded by Jiangsu Provincial Key Research and Development Program of China (BE2015066).

\section{References}

[1] G. Li, H. Liu, T. Li, J. Wang, Surface modification and functionalization of silk fibroin fibers/fabric toward high performance applications, Mater. Sci. Eng. C 32 (2012) 627-636.

[2] Y. Zhou, J. Zhang, R.-C. Tang, J. Zhang, Simultaneous dyeing and functionalization of silk with three natural yellow dyes, Ind. Crop. Prod. 64 (2015) 224-232.

[3] G. Li, H. Liu, H. Zhao, Y. Gao, J. Wang, H. Jiang, R.I. Boughton, Chemical assembly of $\mathrm{TiO}_{2}$ and $\mathrm{TiO}_{2} @$ Ag nanoparticles on silk fiber to produce multifunctional fabrics, J. Colloid Interf. Sci. 358 (2011) 307-315.

[4] L. Budama, B.A. Çakır, Ö. Topel, N. Hoda, A new strategy for producing antibacterial textile surfaces using silver nanoparticles, Chem. Eng. J. 228 (2013) 489-495. 